\begin{tabular}{l|l} 
Revista Educación \\
ISSN: 0379-7082 \\
ISSN: 2215-2644 \\
revedu@gmail.com \\
Universidad de Costa Rica \\
Costa Rica
\end{tabular}

\title{
Descubrimiento del estilo de aprendizaje dominante en estudiantes de Matemática Superior
}

Coto Jiménez, Marvin

Descubrimiento del estilo de aprendizaje dominante en estudiantes de Matemática Superior

Revista Educación, vol. 44, núm. 1, 2020

Universidad de Costa Rica, Costa Rica

Disponible en: http://www.redalyc.org/articulo.oa?id=44060092037

DOI: https://doi.org/10.15517/revedu.v44i1.38571

Esta obra está bajo una Licencia Creative Commons Atribución-NoComercial-SinDerivar 3.0 Internacional. 


\title{
Descubrimiento del estilo de aprendizaje dominante en estudiantes de Matemática Superior
}

\author{
Discovering Dominant Learning Styles in Higher Level Math Students
}

Marvin Coto Jiménez

Universidad de Costa Rica, Costa Rica

marvin.coto@ucr.ac.cr

iD http://orcid.org/0000-0002-6833-9938
DOI: https://doi.org/10.15517/revedu.v44i1.38571

Redalyc: http://www.redalyc.org/articulo.oa?id=44060092037

Recepción: 12 Septiembre 2019

Aprobación: 12 Diciembre 2019

\section{Resumen:}

La presente investigación se desarrolló con el objetivo de descubrir cuáles son los estilos de aprendizaje dominantes en estudiantes de Matemática Superior de la carrera de Ingeniería Eléctrica en la Universidad de Costa Rica, entre los años 2018 y 2019. El conocimiento de los estilos de aprendizaje dominante es de utilidad para promover la mejora en los procesos de enseñanza en esta población, y dirigir de forma óptima los esfuerzos por procurar el aprendizaje significativo. El estudio se ejecutó con el siguiente procedimiento: Elaboración del estado del arte sobre estilos de aprendizaje, especialmente en educación superior, para conocer su metodología y alcance. A partir de este, se determinó la conveniencia y viabilidad de aplicar el instrumento propuesto por FelderSilverman, para categorizar a la muestra de 122 estudiantes de acuerdo con su estilo predominante. Posteriormente, se organizó el procesamiento de datos y su análisis, en el cual se destaca la técnica de agrupamiento, para descubrir el estilo de aprendizaje dominante. De acuerdo con las categorías de Felder-Silverman, el estilo de aprendizaje dominante en esta población de estudiantes es de tendencia visual (en un 55,7\% de la población) y sensorial (en un 45,9\% de la población), lo cual es un indicador de la preferencia hacia el aprendizaje basado en datos y procedimientos establecidos, usando materiales concretos y visuales.

Palabras ClaVE: Enseñanza Superior, Formación de ingenieros, Ingeniería eléctrica, Matemática, Proceso de aprendizaje.

\section{Abstract:}

The objective of this study is to discover the dominant learning styles among higher level Math students in the Electrical Engineering Program at the University of Costa Rica during 2018-19. Knowledge of the different dominant learning styles is useful to enhance teaching processes among this population group and effectively target efforts to attain significant learning. The study was executed using the following procedure: elaboration of state of the art on learning styles, especially higher education to learn its scope and methodology. The convenient and viable Felder -Silverman Learning Style Model was also used to classify a sample of 122 students based on their predominant learning style. Afterwards, the data was processed and analyzed through a grouping technique to determine the dominant learning style. According to Felder-Silverman, the dominant learning style in this student population is visualizing ( $55.7 \%$ of the population) and sensing/intuitive ( $45.9 \%$ of the population), which indicate preference towards learning based on data and established procedures using tangible and visual material.

KEYWORDS: Higher Learning, Engineering Training, Electrical Engineering, Mathematics, Learning Processes.

\section{INTRODUCCIÓN}

En el aprendizaje confluyen una gran cantidad de elementos, los cuales forman parte y provienen de quien enseña, de quien aprende y de su entorno. Estos elementos conforman un entramado de relaciones complejas que pueden evolucionar e influir en distinta medida al proceso de aprendizaje.

Dada la cantidad de variables que emergen para modelar el proceso, se hace imposible controlarlas a todas en aras de lograr los mejores resultados para cada caso particular. Sin embargo, es posible seleccionar algunas estrategias que sean adecuadas para contribuir con el desarrollo de conocimientos, competencias y habilidades que les permitan a los alumnos lograr resultados deseados (Montero, Pedroza, Astiz y Vilanova, 2015, p. 89). 
En el caso particular de matemática, existe una importante y activa área de investigación en el campo de su enseñanza, dadas las dificultades que enfrentan muchos estudiantes durante su aprendizaje (Sibaja y Rojas, 2018). Tradicionalmente, los estudios se han enfocado más en niveles básicos e intermedios del currículo, donde la cantidad de estudiantes es mayor que en niveles superiores, y donde, a pesar de los intereses preliminares que pueden mostrar, la educación matemática recibida es usualmente igual para todos. No por esto, el estudio del aprendizaje matemático en niveles superiores deja de tener relevancia, pues el estudiantado enfrenta aún dificultades significativas con diversos contenidos, en cursos de baja promoción o donde el aprendizaje significativo no es promovido en toda su extensión.

Es posible señalar que la investigación sobre los estudiantes, los obstáculos que encaran y cómo impactan en el aprendizaje, es un campo en construcción en América Latina, el cual busca actualmente mayores grados de consolidación a escala teóricametodológica, aplicado en situaciones reales de experiencias de aula (Ventura, Palou, Széliga, y Angelone, 2014, p.179).

A pesar de que no es posible determinar por completo cómo aprende esta población, es posible identificar con claridad que existen diferentes formas de hacerlo, tales como: observando, escuchando, reflexionando, debatiendo, actuando, memorizando, visualizando y construyendo analogías (Camaná, 2017). Por su parte, las y los docentes también aplican diferentes estrategias, como la lectura, el debate, la resolución de ejercicios, la explicación profunda de la teoría, análisis de caso, entre otros.

Como se ha mostrado en distintas investigaciones, tales como de García (2013), Ruiz (2014), Díaz y Sánchez (2017) y Camaná (2017), los estilos de aprendizaje se pueden determinar en estudiantes de educación superior, incluyendo estudiantes de ingeniería, utilizando instrumentos validados en la teoría. La conveniencia de estos estudios ha sido ampliamente discutida y comprobada para mejorar procesos a partir de una caracterización precisa de la población en estos aspectos.

En el presente artículo se presentan los resultados de una investigación elaborada con el objetivo de conocer cuáles son los estilos predominantes de aprendizaje de estudiantes de Matemática Superior de Ingeniería Eléctrica de la Universidad de Costa Rica a partir de un enfoque objetivo, del cual no existe conocimiento a priori. Esto objetivo se sigue con la finalidad de brindar insumos para impactar positivamente la enseñanza de los contenidos, pues del conocimiento del estilo predominante se derivan las estrategias más efectivas para contribuir con el aprendizaje significativo.

\section{ANTECEDENTES}

Uno de los elementos analizados en el proceso de enseñanza aprendizaje es el conocimiento de los estilos de aprendizaje del estudiantado, el cual parte del reconocimiento de que existen diferentes formas de aprender. Esto se refiere a formas variadas de percibir, procesar y comprender los contenidos recibidos en una clase. Como indica Ventura et al. (2014):

Cada estudiante tiene un sistema de normas, nociones, comportamientos e ideas que le dan sentido a la práctica. Para comprender el sentido de esa práctica es necesario analizar el estilo de aprendizaje del estudiante, es decir, cómo recibe y procesa la información (p. 179).

Keefe (1979, citado por Fiallos y Carrera, 2018), señala que los estilos de aprendizaje son "rasgos cognitivos, afectivos y fisiológicos que operan como posibles indicadores de cómo un individuo promedio percibe, apropia e interactúa o responde a una determinada información" (p.307). El descubrir los estilos de aprendizaje que caracterizan a un grupo de estudiantes es un factor ampliamente estudiado como insumo para establecer estrategias y actividades que favorezcan los estilos predominantes. Por ejemplo, si se conoce un estilo de aprendizaje dominante, se pueden rediseñar las dinámicas de clase, de presentación de contenidos y de evaluación para favorecer el aprendizaje significativo. 
En América Latina, las ventajas que puede brindar este conocimiento para los procesos de enseñanza aprendizaje se ha plasmado en estudios como el de Tocci (2015), desarrollado para una población de estudiantes de ingeniería. En Costa Rica no se encuentran trabajos de esta naturaleza sobre poblaciones de estudiantes de Ingeniería Eléctrica, por lo que la elaboración de la presente investigación puede equiparar los hallazgos y utilidades de las referencias efectuadas fuera del país. Por ejemplo, para analizar la pertinencia de las estrategias actuales de enseñanza o de los materiales didácticos con que se cuenta.

El contexto de este estudio es el curso Matemática Superior, analizado en la presente investigación, este pertenece al bloque común de la carrera de Ingeniería Eléctrica de la Universidad de Costa Rica, es decir, se encuentra entre los cursos que deben ser completados por la totalidad de estudiantes que se encuentren empadronados en esta carrera. El objetivo principal del curso es: Estudiar las herramientas matemáticas más frecuentemente utilizadas para el análisis teórico de la respuesta de sistemas eléctricos lineales invariantes con el tiempo ante diferentes señales eléctricas de entrada.

Los contenidos incluyen herramientas matemáticas utilizadas en sistemas de ingeniería eléctrica, principalmente: transformadas integrales (Transformadas de Laplace y de Fourier), una transformada discreta (Transformada Z), Métodos Numéricos y las bases matemáticas de variable compleja. El curso de desarrolla a lo largo de dieciséis semanas, con cinco horas de clases cada una, distribuidas en lecciones de tres y dos horas según el día correspondiente a las dos sesiones semanales.

Tradicionalmente, la mayoría de los contenidos son desarrollados de una forma procedimental, para aprender a aplicar las transformaciones y procedimientos diversos en determinadas señales. La evaluación se basa principalmente en exámenes donde se pone a prueba la capacidad del estudiantado para aplicar las herramientas matemáticas, en lo cual representa un peso importante las operaciones matemáticas en sí. Los ejercicios de evaluación usualmente tienen encabezados como: Determine la transformada de..., o Calcule la transformada inversa de...

En algunos de los aspectos del curso, es posible establecer una relación con lo indicado por Montero et al. (2015), para el caso particular de métodos numéricos, el cual forma parte de los contenidos de Matemática Superior:

[L]os estudiantes no solo experimentan dificultades para la comprensión y aplicación de los algoritmos numéricos, sino que reducen el aprendizaje de estos procedimientos a procesos rutinarios basados en la mecanización y la memorización, minimizando el razonamiento lógico, el pensamiento creativo, la búsqueda de soluciones, la crítica y el procesamiento y análisis de la información (p. 89)

Características semejantes han sido señaladas también en otras universidades latinoamericanas con respecto a la enseñanza de la ingeniería, como se señala en Tocci (2015). Estas observaciones han puesto de manifiesto la necesidad de diagnosticar y rediseñar los procesos de aprendizaje.

Dado que el aprendizaje significativo de las herramientas matemáticas contempladas en el curso requiere más que la mecanización y memorización señalada anteriormente, es de importancia avanzar hacia la creación de recursos y experiencias didácticas que lo favorezcan en mayor medida. Como es sabido, el alumnado tiene diferentes formas de aprender y asimilar, en ocasiones privilegiando el uso de algunos sentidos, como ha sido estudiado ampliamente por la psicología y ciencias de la educación (Franzoni, Assar, Defude y Rojas, 2008). El reconocimiento e identificación de estas formas de aprender se sintetiza en el concepto de estilos de aprendizaje, desarrollado en la siguiente sección.

\section{Referentes TEÓRICOS}

El término estilo de aprendizaje hace referencia a que existe una variedad de formas en las cuales cada estudiante aprende, las cuales pueden ser identificadas y aprovechadas con fines de mejorar el aprendizaje. Entre los rasgos que intervienen en determinar los estilos se encuentran el cognitivo (forma en que se 
logran estructurar los contenidos), el afectivo (motivaciones, experiencias) y el fisiológico (género y aspectos biológicos) (Fiallos y Carrera, 2018).

Sibaja y Rojas (2018) apuntan que otros términos como Modelos de enseñanza y Estilos de enseñanza se utilizan como sinónimos en diversas investigaciones, las cuales reflexionan y evalúan los modelos o estilos de enseñanza para mejorar las dinámicas de aula.

En educación, este concepto ha sido de particular importancia, como señalan Ojeda, y Herrera (2013):

A diferencia de los estudios acerca de los estilos cognitivos, las investigaciones acerca los estilos de aprendizaje encontraron en el ámbito de la educación mejores condiciones para su aplicación ya que se refiere a la forma y manera en que se realiza la terea partiendo del ser individual (p. 163).

De acuerdo con Tapias, Cué, Vivas y Rincón (2011), existen una gran cantidad de instrumentos que miden los estilos de aprendizaje. Entre estos instrumentos se destaca el modelo de estilos de aprendizaje de FelderSilverman, debido a su aplicación extendida y al uso de instrumentos de medición validados, que proveen la identificación de estilos de aprendizaje en categorías específicas (Felder y Spurlin, 2005). Por ejemplo, en América Latina ha sido aplicado recientemente para poblaciones de estudiantes de ingeniería en estudios como el de Tocci (2015), Puello, Fernández y Cabarcas (2014) y Ocampo, Guzmán, Camarena y de Luna (2014). En cuanto a la fiabilidad del instrumento, un estudio de Brito y Espinosa (2014) con 459 estudiantes de medicina de la Universidad Autónoma de San Luis Potosí, México, mostró índices de fiabilidad entre 0,38 y 0,61 en el instrumento. Por su parte, en un trabajo de Hosford y Siders (2010) se evaluaron 340 estudiantes de diferentes carreras de la Greek University en Grecia, y se determinaron valores de alfa de Cronbach aceptables para cada dimensión del cuestionario de Felder-Silverman.

De manera que la aplicabilidad y confiabilidad estadística de este instrumento ha sido probada ampliamente en diversos estudios, tanto relacionados con ingeniería como con otras áreas de la educación superior.

En este modelo de Felder-Silverman, se establecen cuatro dimensiones de estilos de aprendizaje: uno relacionado con el procesamiento (categorizado como activo o reflexivo), uno con la percepción (categoría sensorial o intuitiva), uno con la representación (ya sea visual o verbal) y una con la compresión (secuencial o global).

Las características generales de cada individuo en cada uno de estos ejes son descritas de la siguiente manera, de acuerdo con Ventura et al. (2014):

- Estilos sensorial e intuitivo. Las personas sensoriales prefieren aprender contenidos como hechos, casos y datos, y resolver problemas con métodos establecidos. Los intuitivos prefieren descubrir relaciones y construir nuevos conceptos. Tienen gusto por la innovación y el cambio, y no son afines a la repetición mecánica de procedimientos.

- Estilos activo y reflexivo. Las personas activas tienden a retener y comprender mejor la información cuando la manipula mediante la discusión, aplicación, experimentación u otras actividades propias del trabajo con grupos. Las reflexivos prefieren pensar primero silenciosamente antes de ejecutar acciones o tomar decisiones, y prefieren trabajar en forma individual.

- Estilos visuales y verbales. Las personas visuales recuerdan mejor lo que ven si se trata de materiales figurativos. Las verbales recuerdan más lo que escuchan y las explicaciones representadas con la palabra escrita.

- Estilos secuencial y global. Las personas secuenciales prefieren aprender mediante un orden de progresión paso a paso desde lo particular hacia lo general. Las personas globales comprenden aspectos generales y relaciones entre temas y luego profundizan en los aspectos particulares de cada contenido (p.181). 
Entre los estudios recientes en áreas afines a la Ingeniería Eléctrica donde han sido analizados los estilos de aprendizaje con la prueba de Felder-Silverman fuera de la región latinoamericana se encuentran los de Kannapiran, Kob, Rus y Sulaiman (2018), para el caso de Ingeniería Mecánica en Premier Polytechnic Malaysia, y Fang, bin Daud, Al Haddad y Mohd (2017) en la misma carrera de Universiti Teknologi Malaysia. También Chan, Shamsuddin y Suratkon (2018) para el caso de Ingeniería Civil también en Malasia ( Universiti Tun Hussein Onn Malaysia).

Para examinar los resultados de la prueba en diversas poblaciones, se han utilizado métodos como la clasificación a través de centroides (Díaz, Rico, Arellano, y Guzma\#n, 2015), así como el análisis mediante técnicas estadísticas descriptivas (Camaná, 2017).

Estos análisis se complementan, dado que en el análisis de cuatro ejes establecido a partir del test de FelderSilverman, es posible analizar cada uno individualmente (con las técnicas descriptivas) para determinar aquellos que son más dominantes, o bien marcar una tendencia de la población de forma global, estableciendo un punto alrededor del cual se agrupa la mayor parte de la muestra (con el análisis de centroides). De esta manera, ambos análisis brindan posibilidades de describir y dar información de los ejes, de forma individual o en conjunto.

\section{Procedimientos metodológicos}

\section{Muestra}

En este estudio participaron voluntariamente 122 estudiantes de la carrera de Ingeniería Eléctrica de la Universidad de Costa Rica, en la Sede Rodrigo Facio. El curso forma parte del cuarto bloque semestral del programa de Bachillerato y Licenciatura en Ingeniería Eléctrica. Es decir, de acuerdo con la malla curricular, se trata de un curso de mitad de carrera para obtener el grado de bachillerato (plan de cuatro años). Este curso se considera troncal, pues la totalidad de estudiantes de la carrera, en cualquiera de los énfasis que se ofrecen (Computadoras y Redes, Electrónica y Telecomunicaciones, Sistemas de Energía) debe aprobarlo para obtener el grado. Los voluntarios que completaron el instrumento de medición provenían de dos semestres consecutivos del curso. Un total de 122 estudiantes participaron, de la población anual de 150, es decir, la participación voluntaria fue de un $81,34 \%$.

\section{Instrumento de medición}

El instrumento utilizado para la recolección de datos fue el Índice de Estilos de Aprendizaje, de FelderSilverman. De acuerdo con este modelo, el estilo de aprendizaje se puede medir según cuatro dimensiones: el procesamiento, la percepción, la representación y la comprensión (Ventura et al., 2014). Cada dimensión está compuesta por dos estilos de aprendizaje opuestos: activo-reflexivo (procesamiento); sensorial-intuitivo (percepción); visual-verbal (representación); secuencial-global (comprensión).

El instrumento desarrollado para medir estos estilos de aprendizaje, utilizado ampliamente en las referencias mencionadas en los referentes teóricos, consta de 44 preguntas en las cuales el alumnado escoge entre dos opciones excluyentes, indicadas como A o B. Cada una de las respuestas se contabiliza de acuerdo con el correspondiente estilo definido por el instrumento.

Cuando se tienen todas las respuestas, se ejecuta una suma algebraica de los puntajes obtenidos en cada eje, y de esta manera se pueden establecer niveles de preferencia: preferencia alta, preferencia media y equilibrio entre cada uno. 


\section{Procedimiento}

El procedimiento general se ilustra en la Figura 1. El instrumento de Felder-Silverman se implementó en un formulario de Google. Por medio de un enlace, se invitó a los participantes a completarlo, utilizando redes sociales y la plataforma de Mediación Virtual de la Universidad de Costa Rica.

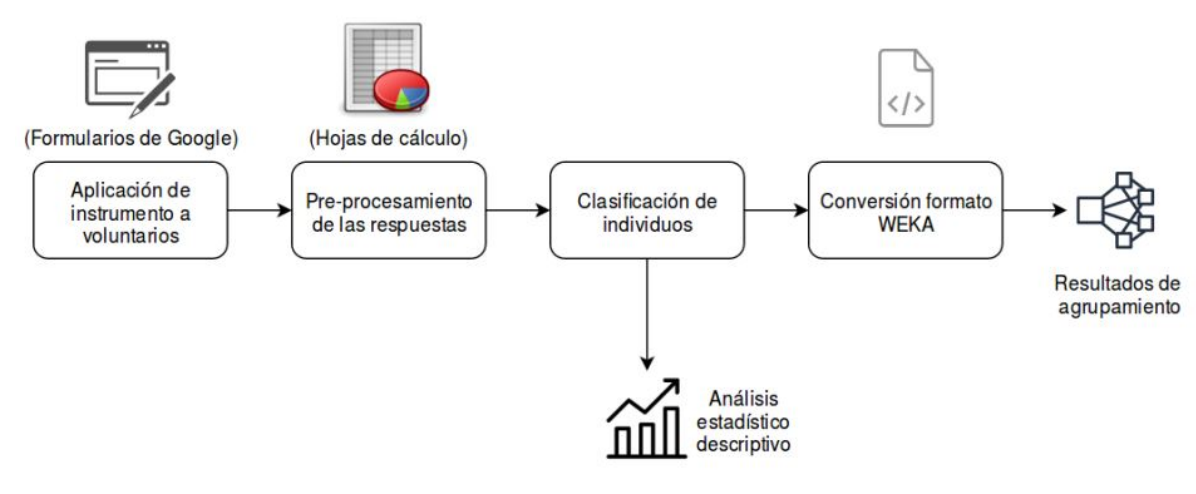

FIGURA 1

Esquema del procedimiento seguido para obtener y procesar la información

Fuente: Elaboración propia (2019)

Una vez que se recabaron las respuestas, se procedió a su pre-procesamiento, al utilizar la escala del instrumento para determinar la predominancia de cada estudiante en cada uno de los ejes. Con esto fue posible desarrollar el análisis estadístico descriptivo de cada eje.

Para el análisis de agrupamiento, manualmente se efectuó la adaptación de los datos al formato requerido por el software Weka para la aplicación del algoritmo de agrupamiento.

\section{ANÁLISIS Y DISCUSIÓN DE RESULTADOS}

Para el análisis de información se utilizaron herramientas semejantes a las propuestas por Fiallos y Carrera (2018), así como Camaná (2017), por lo cual se utilizó el software de análisis de datos Weka, específicamente con la técnica de FarthestFirst para determinar los centroides de la población de acuerdo con sus respuestas al instrumento de Felder-Silverman. Tal como se ilustra en estas referencias, esta técnica permite separar a la muestra de estudiantes en grupos característicos y establecer el centroide, o caso característico, en cada uno. Con este centroide se puede caracterizar a los grupos en que se divide la muestra, y así dar un descriptor de los cuatro ejes del instrumento para cada uno de los grupos que se establezcan en la muestra.

\section{Predominancia de cada eje}

En la muestra analizada, se estudió cada eje para describir la población en términos de la predominancia de cada uno de forma separada. En la Figura 2 se muestran los resultados del primer eje. Es notoria la predominancia del equilibrio en la muestra de estudiantes, incluso sobre el total de participantes con predominancia activa y reflexiva. 


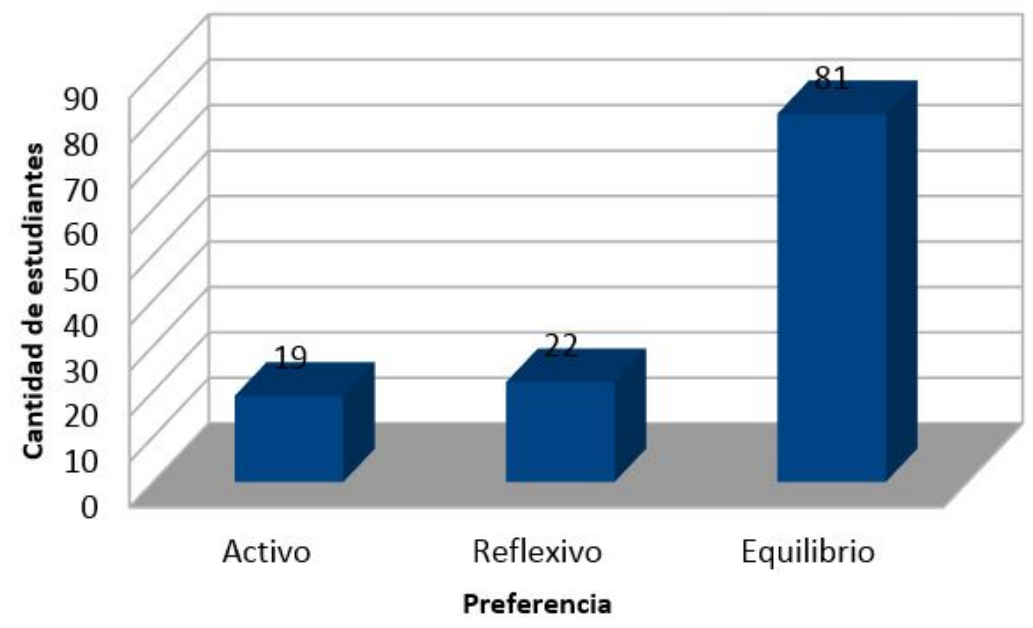

FIGURA 2

Resultados de evaluación del primer eje: Activo-Reflexivo

Elaboración propia (2019)

En otros estudios desarrollados sobre poblaciones de estudiantes de Ingeniería en América Latina, también se ha mostrado una preferencia sobre el eje sensorial en relación con el intuitivo. Por ejemplo, en el estudio de Tocci (2015), donde se analizan varios énfasis de esta carrera en Argentina, se reporta un equilibrio en el eje equilibrio activo-reflexivo. Por su parte, Puello et al. (2014), reportan una preferencia marcada hacia el perfil activo, en el caso de estudiantes de Ingeniería de Sistemas en Colombia. El resultado de equilibrio entre ambos ejes en el presente estudio es coincidente con los hallazgos de Ocampo et al. (2014) para estudiantes de Ingeniería en Ciencias Computacionales, en México.

La predominancia del estilo sensorial sobre el intuitivo es muy notoria, como se muestra en la Figura 3. Es destacable que el estilo intuitivo está presente solo en una minoría de la muestra (un 9\%), mientras que la predominancia sensorial está presente en un $45,9 \%$ del total.

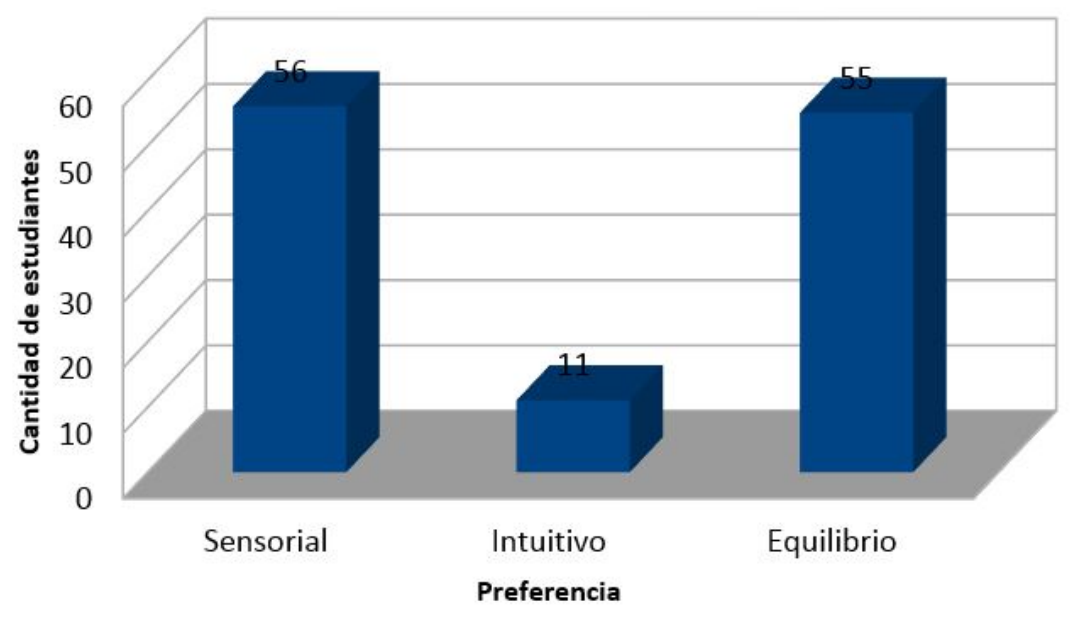

FIGURA 3

Resultados de evaluación del segundo eje: Sensorial - Intuitivo Elaboración propia (2019) 
En el estudio de Tocci (2015) se reporta una preferencia moderada hacia lo sensorial. Los resultados del presente estudio son un poco más marcados hacia esta preferencia, en concordancia con el estudio de Puello et al. (2014) para el caso de estudiantes de Ingeniería de Sistemas. De igual manera, la preferencia sensorial es más fuerte que la reportada por Ocampo et al. (2017) en la población de estudiantes correspondiente.

En cuanto al eje visual-verbal, como se muestra en la Figura 4, la predominancia a visual es clara, con un $55,7 \%$ de la muestra. La predominancia verbal es solamente de un $9 \%$.

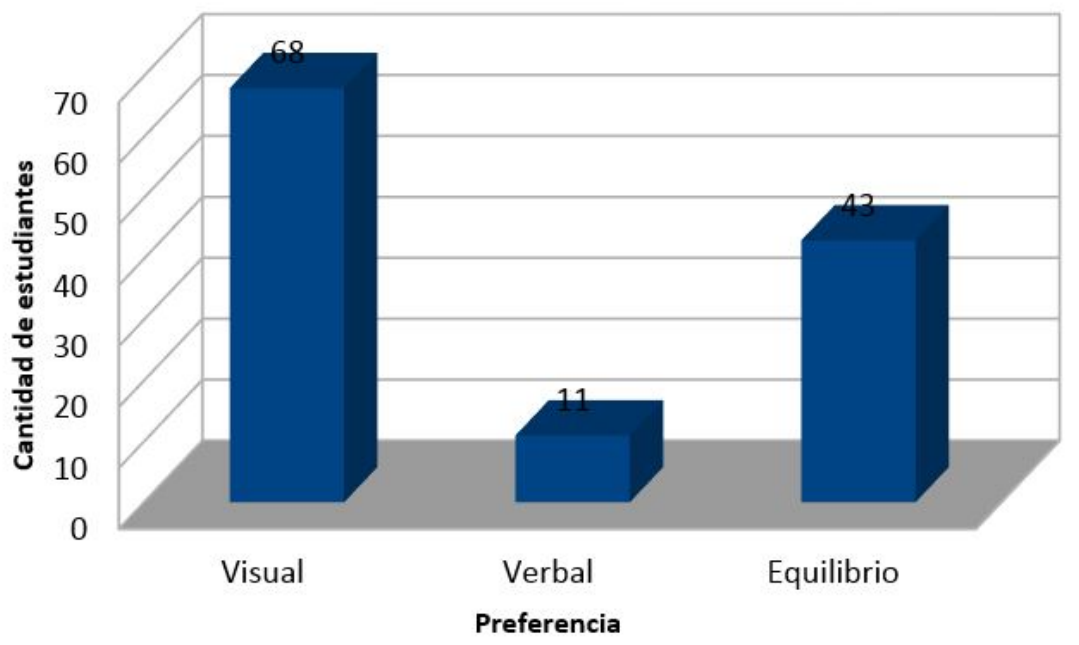

FIGURA 4

Resultados de evaluación del tercer eje: Visual-Verbal

Fuente: Elaboración propia (2019)

Tanto en el estudio de Tocci (2015) como el de Puello et al. (2014) y de Ocampo et al. (2017) también se encuentra una preferencia de lo visual sobre lo verbal en estudiantes de Ingeniería, en coincidencia con la muestra analizada en Ingeniería Eléctrica.

Finalmente, según la Figura 5,en el eje secuencial-global, no hay una predominancia marcada, sino que la mayoría de estudiantes (un 69,7\%) presenta equilibrio entre ambos. Sin embargo, es de destacar que la predominancia global está presente en solamente un 6,6\% de la muestra. 


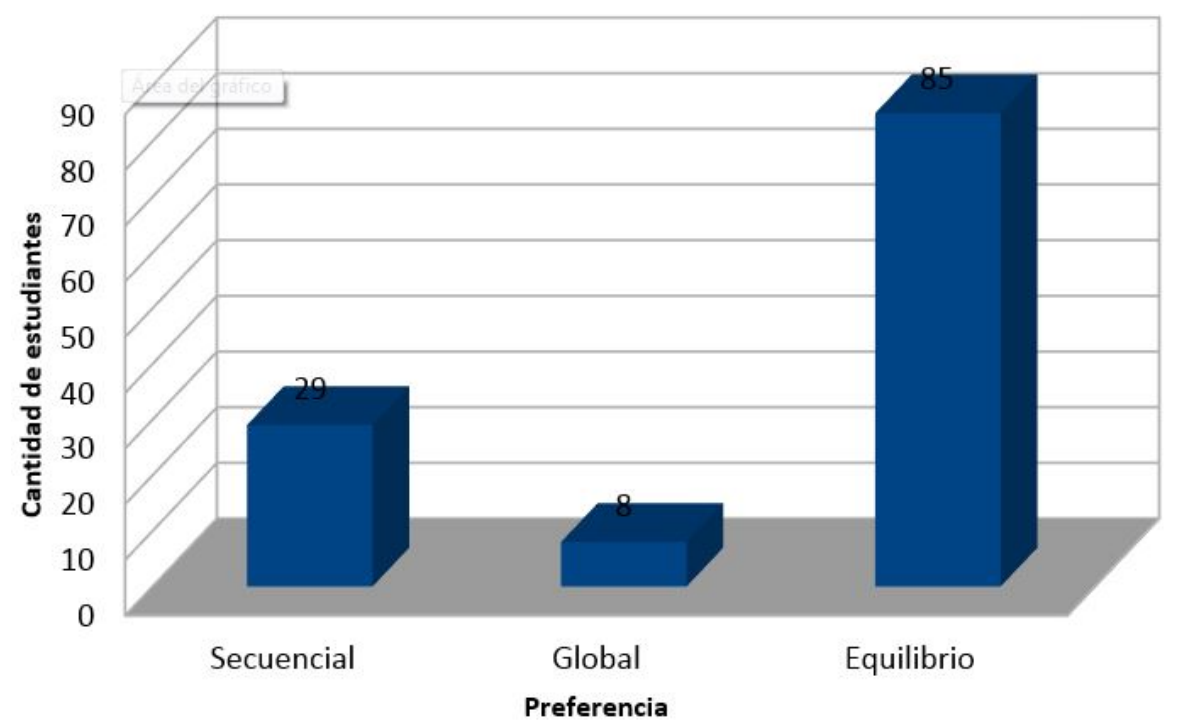

FIGURA 5

Resultados de evaluación del cuarto eje: Secuencial - Global

Fuente: Elaboración propia (2019)

En el estudio de Puello et al. (2014), la preferencia secuencial es menos marcada que la global, en relación a las preferencias de los otros ejes, de forma semejante a Tocci (2015) y Ocampo et al. (2017), quien determina una preferencia hacia lo secuencial solamente de forma moderada.

De manera que, en cuanto a ejes individuales, la muestra tiene mayores tendencias en sensorial-intuitivo y en visual-verbal, y es mayoritariamente coincidente con otras poblaciones de estudiantes de ingeniería en la región. Esta coincidencia puede considerarse un indicador de una predominancia de estilo de aprendizaje previo a la elección de la carrera de ingeniería, aunque este aspecto debe profundizarse con mayores estudios. Por otra parte, la predominancia de estilos puede correlacionarse con otros estudios posteriores en aspectos como rendimiento académico, y de esta manera fortalecer aquellos aspectos que beneficien los procesos de aprendizaje de grupos específicos de estudiantes.

\section{Análisis de agrupamiento}

El análisis de agrupamiento permite determinar una tendencia general de la muestra alrededor de una valoración de todos los ejes a la vez. Como observa Camaná (2017), un análisis de este tipo se efectúa "con el fin de identificar grupos homogéneos dentro de los estudiantes encuestados” (p. 202). De esta manera, con un algoritmo como FarthestFirst, se establecen grupos con un centroide, que constituiría un caso explicativo para cada agrupamiento. Los grupos se establecen a partir de la mínima distancia (o diferencia) entre las respuestas de cada participante y las del centroide o caso representativo.

En la Figura 6 se muestra el resultado del algoritmo seleccionado, aplicado con el programa Weka, desarrollado para aplicar distintos algoritmos relacionados con problemas de agrupamiento y clasificación a conjuntos de datos sujetos de este tipo de análisis. Lo que se indica como Cluster 0 y Cluster 1 se refiere a aquellos conjuntos de respuestas sobre los que se agrupan las respuestas globales de toda la muestra. En otras palabras, son los dos casos alrededor de los cuales se agrupa la totalidad de la muestra.

Cada caso corresponde a una secuencia de respuestas del test (cada respuesta es opción A o B), y permiten establecer dos grupos distinguibles en esta muestra. La secuencia de respuestas de cada grupo corresponde a los siguientes resultados: 
- Grupo 1 (85\% de la muestra):

- Eje activo - reflexivo: Equilibrio

- Eje sensorial - intuitivo: Preferencia sensorial

- Eje visual - verbal: Equilibrio

- Eje secuencial - global: Equilibrio

- Grupo 2 (15\% de la muestra):

- Eje activo - reflexivo: Equilibrio

- Eje sensorial - intuitivo: Preferencia intuitivo

- Eje visual - verbal: Preferencia visual

- Eje secuencial - global: Preferencia global

Esta información complementa los resultados de la sección anterior, en cuanto a predominancia de equilibrio en tres de los ejes del grupo mayoritario, mientras que se encuentra uno con preferencia hacia lo sensorial. En el segundo agrupamiento un $15 \%$ de los participantes es afín a la preferencia intuitiva, visual y global, mientras que en el eje activo-reflexivo la tendencia es al equilibrio.

De esta manera, se puede caracterizar a la gran mayoría de esta población con clara preferencia sensorial, como el eje diferenciador de estilo de aprendizaje. Otros aspectos, como el aprendizaje de lo general a lo específico o viceversa, no se consideran relevantes en la característica poblacional para impactar la práctica docente o los materiales didácticos. La tendencia al equilibrio en los ejes de Felder-Silverman contrasta con tendencias más marcadas hacia preferencias en otras poblaciones de ingeniería, como las reportadas por Ocampo et al. (2014), donde población de estudiantes de Ingeniería tienen clara preferencia hacia los ejes activo y secuencial.

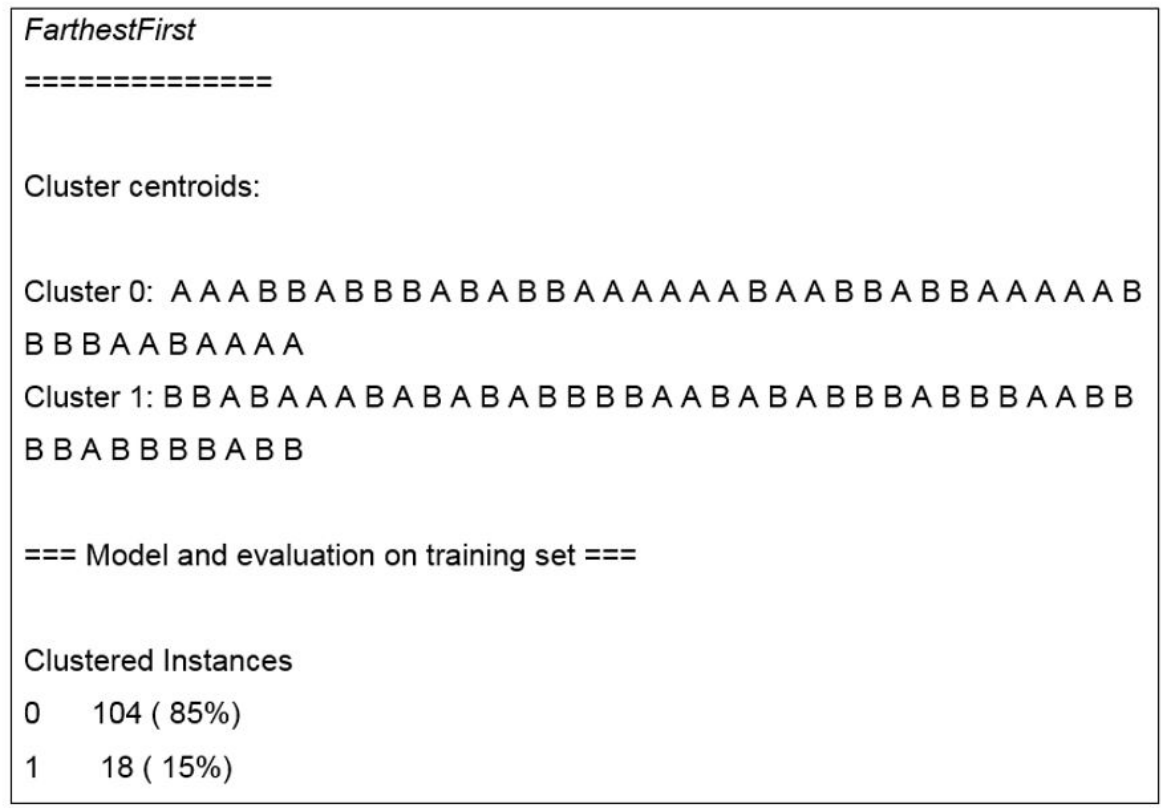

FIGURA 6

Resultados mostrados por el programa Weka

Fuente: Elaboración propia (2019) 


\section{Conclusiones}

En el presente artículo se presentaron los antecedentes, instrumentos y procedimientos con los cuales se determinó el estilo de aprendizaje predominante en estudiantes del curso Matemática Superior de la Escuela de Ingeniería Eléctrica de la Universidad de Costa Rica. El instrumento aplicado fue la prueba de FelderSilverman. Tanto el análisis de cada uno de los ejes individuales de este instrumento, como en análisis de agrupamiento desarrollado denota que el estudiantado tiende a un equilibrio en los ejes ( $80 \%$ en activoreflexivo y del $70 \%$ en secuencial-global), pero hay tendencias marcadas hacia la preferencia visual (55,7\%) y sensorial $(45,9 \%)$ como estilos de aprendizaje predominantes. Este hallazgo de estilos de aprendizaje asemeja las características de esta población de Ingeniería Eléctrica con otras especialidades de la Ingeniería analizadas en América Latina.

La coincidencia entre estas poblaciones puede tomarse como indicador de una tendencia general de quienes optan por ejecutar estudios superiores en esta disciplina, e incluso como un indicador vocacional para aspirantes a seguir estas carreras.

Por otra parte, la información de estilos de aprendizaje dominante brinda insumos para orientar las prácticas docentes y los materiales a utilizar en el curso de Matemática Superior, los cuales deben tener características que sean afines a estos estilos predominantes, tales como hechos, casos y datos (por el eje sensorial) y materiales figurativos (por el eje visual). Finalmente, se pueden abrir nuevas posibilidades para relacionar los resultados con rendimiento académico, género y percepción del curso Matemática Superior u otros de la carrera de Ingeniería Eléctrica por parte de las y los estudiantes.

\section{REFERENCIAS}

Brito, M. D., y Espinosa, R. (2015). Evaluación de la fiabilidad del cuestionario sobre estilos de aprendizaje de Felder y Soloman en estudiantes de medicina. Investigación en educación médica, 4(13), 28-35. doi: https:// doi.org/10.1016/S2007-5057(15)72166-6

Camaná, R. (2017). Descubrimiento del estilo de aprendizaje dominante de estudiantes de la carrera de tecnología en análisis de sistemas. In Crescendo, 8(2), 193-209.

Chan, C. M., Shamsuddin, A., y Suratkon, A. (2018). Of Grades, Activities and Learning Styles: Correlation in a Civil Engineering Technology Course. Advanced Science Letters, 24(6), 4576-4580. doi: https://doi.org/10.1166/ asl.2018.11660

Díaz, M.A., y Sánchez, I.O. (2017). Caracterización de estilos de aprendizaje en estudiantes de ingeniería de jornadas diurnas y vespertinas. Enseñanza \& Teaching: Revista Interuniversitaria de Didáctica, 35(2), 97-108. doi: http:// dx.doi.org/10.14201/et201735297108

Díaz, C.O., Rico, A.K., Arellano, A., y Guzma\#n, A. (2013). Estrategia para detectar Estilos de Aprendizaje usando la técnica de particiones. Revista de Estilos de Aprendizaje, 6(12),1-14.

Fang, N., bin Daud, M. F., Al Haddad, S. A., y Mohd, K. (2017). A quantitative investigation of learning styles, motivation and learning strategies for undergraduate engineering students. Global Journal of Engineering Education, 19(1), 4-29.

Felder, R.M., y Spurlin, J. (2005). Applications, reliability and validity of the index of learning styles. International journal of engineering education, 21(1), 103-112.

Fiallos, R. G., y Carrera, R. A. (2018). Descubrimiento del estilo de aprendizaje dominante de estudiantes de la carrera de Tecnología en Análisis de Sistemas. Revista Educación, 42(2), 306-317. doi: https://doi.org/10.15517/ revedu.v42i2.26473

Franzoni, A. L., Assar, S., Defude, B., y Rojas, J. (julio 2008). Student learning styles adaptation method based on teaching strategies and electronic media. En 2008 Eighth IEEE International Conference on Advanced Learning Technologies. Conferencia llevada a cabo en Santander, Cantabria, España. doi: 10.1109/ICALT.2008.149 
García, J.A. (2013). Reflexiones sobre los estilos de aprendizaje y el aprendizaje del cálculo para ingeniería. Revista Electrónica Actualidades Investigativas en Educación, 13(1), 1-28.

Hosford, C.C., y Siders, W.A. (2010). Felder-Soloman's index of learning styles: Internal consistency, temporal stability, and factor structure. Teaching and learning in medicine, 22(4), 298-303.

Kannapiran, S., Kob, C.G., Rus, R. C., y Sulaiman, N.L. (2018). Perception of Mechanical Engineering Students According to a Subject on Felder Silverman Learning Styles. Development, 7(4), 1-12.

Montero, Y.H., Pedroza, M.E., Astiz, M.S., y Vilanova, S.L. (2015). Caracterización de las actitudes de estudiantes universitarios de Matemática hacia los métodos numéricos. Revista electrónica de investigación educativa, 17(1), 88-99.

Ocampo, F., Guzmán, A., Camarena, P., y de Luna, R. (2014). Identificación de estilos de aprendizaje en estudiantes de ingeniería. Revista mexicana de investigación educativa, 19(61), 401-429.

Ojeda, A. F. O., \& Herrera, P. J. C. (2013). Estilos de aprendizaje y rendimiento académico en estudiantes de ingeniería en México. Journal of Learning Styles, 6(11), 160-177.

Puello, P., Fernández, D., y Cabarcas, A. (2014). Herramienta para la Detección de Estilos de Aprendizaje en Estudiantes utilizando la Plataforma Moodle. Formación universitaria, 7(4), 15-24.

Ruiz, Y.P. (2014). Estilo de aprendizaje predominante en los estudiantes del segundo año de Ingeniería Forestal de la Universidad de Guantánamo. EduSol, 14(49), 1-8.

Sibaja, A.E., y Rojas, O.R. (2018). Análisis factorial de correspondencias: Estudio en creencias y estilos de enseñanza en docentes de matemática. Revista Educación, 42(2), 598-628. doi: https://doi.org/10.15517/revedu.v42i2.28911

Tapias, M.G., Cué, J.L., Vivas, M., y Rincón, J. A. S. (2011). Estudio comparativo de los estilos de aprendizajes del alumnado que inicia sus estudios universitarios en diversas facultades de Venezuela, México y España. Revista de estilos de aprendizaje, 4(7), 1-28.

Tocci, A. M. (2015). Caracterización de perfiles de estilos de aprendizaje en alumnos de Ingeniería según el modelo de Felder y Silverman. Journal of Learning Styles, 8(16) 101-118.

Ventura, A. C., Palou, I., Széliga, C. N., y Angelone, L. M. (2014). Estilos de aprendizaje y enseñanza en ingeniería: Una propuesta de educación adaptativa para primer año. Revista Educación en Ingeniería, 9(18), 178-189.

\section{BY-NC-ND}

